\title{
Characterisation, calibration and comparison by international collaborative study of international standards for the calibration of therapeutic preparations of $\mathrm{FSH}$
}

\author{
M P Rose and R E Gaines-Das ${ }^{1}$ \\ Division of Endocrinology and ${ }^{1}$ Informatics Laboratory, National Institute for Biological Standards and Control, Blanche Lane, South Mimms, Potters Bar, \\ Hertfordshire EN6 3QG, UK \\ (Requests for offprints should be addressed to M P Rose)
}

\begin{abstract}
Therapeutic preparations of FSH, used primarily for treatment of infertility, are calibrated by in vivo bioassay against international standards (IS) derived from different sources deemed appropriate to their use according to pharmacopoeial monographs. Menotrophins, which have been used for several decades to treat infertility, have been calibrated against the IS for urinary FSH and LH (ISU) but are now being replaced by highly purified urinary FSH or rDNA-derived FSH (rFSH).

The aim of this study was to evaluate two preparations of human $\mathrm{rFSH}$ and one preparation of highly purified urinary FSH as candidate WHO IS for bioassay in an international collaborative study by 27 laboratories in 12 countries, and to characterise them in a range of in vitro bioassays and immunoassays.

The biological activity of the three candidate standards was confirmed by all laboratories using all assays contributed to the study. Dose-response relationships by in vivo bioassay for any of the candidate standards did not differ significantly from that for the ISU. Dose-response relationships obtained in in vitro bioassays and immunoassays were also broadly similar among these preparations although dose-response lines for some preparations
\end{abstract}

appeared to be non-parallel in some immunoassays. For each of the three candidate IS, estimates of the relative potency in terms of ISU by in vivo bioassay did not differ significantly between laboratories. In contrast estimates by immunoassays and in vitro bioassays showed significant differences between laboratories. Estimates of relative potency of the highly purified candidate IS materials in terms of one another exhibited less inter-laboratory variability than estimates in terms of ISU. Each of the candidate standards showed adequate stability to serve as an IS.

On the basis of the results of this study rFSH (code 92/642) was established as the first IS for FSH, human, recombinant for bioassay with an assigned unitage of 138 IU per ampoule and urinary FSH (code 92/512) was established as the first IS for FSH, human, urinary (urofollitropin) for bioassay with an assigned unitage of 121 IU per ampoule, based on their respective calibration by in vivo bioassay in terms of ISU. These assignments of unitage maintain continuity of unitage for preparations in therapeutic use and also appear to be consistent with one another.

Journal of Endocrinology (1998) 158, 97-114

\section{Introduction}

Follicle-stimulating hormone (FSH) is a heterodimeric, heterogeneous glycoprotein hormone which has been identified in most vertebrate species. It has a central role in reproduction and hence is widely used in human (and animal) reproductive medicine both as a therapeutic agent and as a diagnostic analyte. The potency of human urinary gonadotrophins (menotrophins), used for several decades as therapeutic products in reproductive medicine, is defined by their activity in in vivo bioassay expressed as international units (IU) in terms of the international standard (IS) for urinary FSH and luteinising hormone (LH) (ISU) (WHO Expert Committee on Biological Standardization
1964, 1975, Storring et al. 1976). The relative impurity (Giudice et al. 1994) of these materials necessitates the use of bioassays for determining the potency of their dosage forms (Storring 1988).

Advances in biotechnology have enabled the development of highly purified preparations of urinary-derived and rDNA-derived FSH (rFSH) (De Boer \& Mannaerts 1990, Van Wezenbeek et al. 1990, Hugues 1994) which have been used successfully in the treatment of infertility (Devroey et al. 1992, Howles et al. 1994). However, the origin, purity and structural features (Hard et al. 1990, Amoresano et al. 1996, Harris et al. 1996) of $\mathrm{rFSH}$ differ from those of naturally derived preparations. 
Table 1 Ampouled preparations distributed for analysis

\section{Material}

\section{Ampoule code}

92/510

$92 / 642$

$92 / 512$

$71 / 223$

$71 / 264$

Accelerated thermal degradation samples

Accelerated thermal degradation samples

Accelerated thermal degradation samples
Candidate standard for human $\mathrm{rFSH}$

Candidate standard for highly purified urinary FSH

$$
2^{\text {nd }} \text { ISU }
$$

$3^{\text {rd }}$ ISU

$92 / 510$ stored at $+20^{\circ} \mathrm{C},+37^{\circ} \mathrm{C},+45^{\circ} \mathrm{C}$ for 717 or 877 days

$92 / 642$ stored at $+20{ }^{\circ} \mathrm{C},+37{ }^{\circ} \mathrm{C},+45{ }^{\circ} \mathrm{C}$ for 570 or 730 days

$92 / 512$ stored at $+20{ }^{\circ} \mathrm{C},+37^{\circ} \mathrm{C},+45^{\circ} \mathrm{C}$ for 392 or 552 days
Nominal content

$10 \mu \mathrm{g}$ human $\mathrm{rFSH}$

2 mg human plasma albumin

$10 \mathrm{mg}$ mannitol

$15 \cdot 4 \mu \mathrm{mol} \mathrm{NaCl}$

$10 \mu \mathrm{g}$ human $\mathrm{rFSH}$

2 mg human plasma albumin

$10 \mathrm{mg}$ mannitol

$15 \cdot 4 \mu \mathrm{mol} \mathrm{NaCl}$

$10 \mu \mathrm{g}$ immunoaffinity purified urinary FSH

2 mg human plasma albumin

$10 \mathrm{mg}$ mannitol

$15 \cdot 4 \mu \mathrm{mol} \mathrm{NaCl}$

See reference

(Storring et al. 1976)

As $71 / 223$

As $92 / 510$

As $92 / 642$

As $92 / 512$
We describe in this paper the results of an international collaborative study carried out by 27 laboratories in 12 countries to compare candidate standards of highly purified human urinary FSH and of $\mathrm{rFSH}$ with the ISU.

The aims of the study were to: (1) calibrate urofollitropin in terms of the ISU; (2) assess the need for a standard for $\mathrm{rFSH}$; (3) calibrate $\mathrm{rFSH}$ in terms of the ISU; and (4) assess the stability of candidate IS preparations.

\section{Materials and Methods}

\section{Materials}

Materials distributed for this study are listed in Table 1 and were identified to participants in the study by a letter code only, and except for in vivo bioassays were assumed to have a common nominal potency so that they were all treated as nearly as possible identically. Where assay capacity permitted, two ampoules of the same material but with different codes (coded duplicates) were included to provide an independent assessment of intra-assay variability. For in vivo assays different codes were used, and nominal potencies were given based on the best available information so that in vivo assays using the minimum number of animals were carried out.
Materials evaluated as candidate standards were generously donated by the Ares-Serono Group, Geneva, Switzerland and Organon International B.V., Oss, The Netherlands. The bulk materials arrived at National Institute for Biological Standards and Control (NIBSC) either as an aqueous solution containing $0.83 \mathrm{mg}$ protein per $\mathrm{ml}$ (by the Lowry method using human serum albumin as the standard), or as a lyophilised powder containing $27 \mu \mathrm{g}$ protein (by amino acid analysis) per $\mathrm{mg}$ of excipient $(1 \cdot 54 \%$ sucrose, $0.46 \%$ citrate) which was redissolved in $10 \mathrm{ml}$ water immediately prior to ampouling. These materials were stored at $-20{ }^{\circ} \mathrm{C}$ and $+4{ }^{\circ} \mathrm{C}$ respectively prior to ampouling.

Candidate IS for rFSH and highly purified human urinary FSH These consisted of two batches of ampoules, coded $92 / 510$ and $92 / 642$, containing highly purified human $\mathrm{rFSH}$ produced in Chinese hamster ovary (CHO) cells and a batch of ampoules, coded 92/512, containing a preparation of immunoaffinity-purified human urinary FSH. Samples of ampoules of $\mathrm{rFSH}$ and highly purified urinary FSH which had been stored at elevated temperatures to provide an assessment of stability were also included in the study. Reports describing the production and characterisation of highly purified FSH 
Table 2 List of participants

Dr ME Albertengo, BN Giampaolo \& LM Oliva, Dto. Control de Productos Biológicos, INAME, Av Caseros 2161, 1264 Buenos Aires, Argentina

Dr P Bartolini, Maria Teresa CP Ribela \& Cibele Peroni, IPEN-CNEN/University of Sao Paulo, Travessa R, 400, Cidade Universitária, 05508-900, Sao Paulo, Brazil and Dr S Dalmora, Department of Industrial Pharmacy, Federal University of Santa Maria, RS 97119-900, Brazil

Dr S Benedetto, Sorin Biomedica, Diagnostics Business Unit, Via Crescentino, 13040 Saluggia (VC), Italy

Dr Peter Bodlaender, Technical Director, Diagnostics Products Corporation, 5700 West 96th Street, Los Angeles, CA 90045, USA

Dr Y Combarnous, Director, F Guillon \& I Fontaine, Department of Reproductive Physiology, INRA/CNRS, Nouzilly 37380, France

Dr W de Boer \& Dr R de Leeuw, NV Organon, PO Box 20, 5340 BH Oss, The Netherlands

Dr LM Demers, Director, Clinical Chemistry/Clinical Pathology, Core Endocrine Laboratory, Milton S Hershey Medical Center, The Pennsylvania State University, PO Box 850, Hershey, PA 17033, USA

Dr Roger Duggan, IDS Ltd, Boldon Business Park, Boldon, Tyne \& Wear NE35 9PD, UK

Dr A Eshkol \& Dr G De Luca, Ares Services S.A., 15bis Chemin des Mines, CH-1202 Geneve, Switzerland

The late Mr Keith Ferguson \& Ms Rachel Kennet, Division of Molecular Endocrinology, Department of Chemical Pathology, Hammersmith Hospital, Du Cane Road, London W12 OHS, UK (current contact Dr Richard S Chapman)

Dr SG Hillier, Reproductive Endocrine Laboratory, University of Edinburgh, 37 Chalmers Street, Edinburgh EH3 9EW, UK

Professor AJW Hsueh \& Dr S Minami, Division of Reproductive Biology, Stanford University School of Medicine, Department of Gynecology and Obstetrics, 300 Pasteur Drive, Stanford, CA 94305-5317, USA

Dr Sun Liu-Nan and Dr Xu Li-gen, Chinese Control Laboratory, Ministry of Health, National Institute for the Control of Pharmaceutical and Biological Products, Temple of Heaven, Beijing, China

Professor KP McNatty, Wallaceville Animal Research Centre, PO Box 40063, Upper Hutt, New Zealand

Dr DJ Montague \& Dr AJ Beard, Section Manager, Endocrinology Research and Development, Johnson \& Johnson Clinical Diagnostics Ltd, Pollards Wood Laboratories, Nightingales Lane, Chalfont St Giles, Bucks HP8 4SP, UK

Professor E Nieschlag \& Dr M Simoni, Institute of Reproductive Medicine of the University, Steinfurterstrasse 107, 48149 Munster, Germany

Dr V Padmanabhan, Dr AR Midgley Jr, Ms A Rolfes-Curl, Ms CM Zawacki, Ms M Markovs (Pediatric Endocrine Research), Mr M Kielb \& Ms H Anderson Davis (Reproductive Sciences Program), University of Michigan, CS Mott Children's Hospital, Room D3252 Medical Professional Building, Ann Arbor, MI 48109, USA

Dr David Pelling, BIBRA International, Woodmansterne Road, Carshalton, Surrey SM5 4DS, UK

Dr WR Robertson, Department of Medicine, Section of Clinical Biochemistry, University of Manchester, Hope Hospital, Eccles Old Road, Salford M6 8HD, UK

Dr D Robertson \& Mr PG Burgon, Prince Henry's Institute of Medical Research, PO Box 152, Clayton 3168, Victoria, Australia

Dr Pier Bruno Romelli, R \& D Manager, Technogenetics S.r.l., S.S.N.11 Padana Superiore Km 160, 20060, Vassina de Pecchi (MI), Italy

Dr Matthew Rose, Mrs J Longley \& Mr B Rafferty, Division of Endocrinology, NIBSC, Blanche Lane, South Mimms, Potters Bar, Herts EN6 3QG, UK

Associate Professor L Sjodin \& Mrs K Erlandsson-Persson, Division of Biotechnology, MPA, PO Box 26, S-751 03 Uppsala, Sweden

Dr Jeff Titman, Product Manager, Anagen (UK) Ltd, 4 Spring Lakes Estate, Deadbrook Lane, Aldershot, Hants GU12 4UH, UK

Mr J van As, Euro-Diagnostica B.V., PO Box 2820, 7303 GC Apeldoorn, The Netherlands

Dr Elaine Walker, Molecular Biology Section, Therapeutic Goods Administration Laboratories, PO Box 100, Woden ACT 2606, Australia

Professor Leif Wide, Department of Clinical Chemistry, University Hospital, S-751 85 Uppsala, Sweden 
Table 3 Summary of immunoassays used in the study

Immunoassay type (reported as)

$\begin{array}{cl}\begin{array}{c}\text { Laboratory } \\ \text { number }\end{array} & \\ 1 & \text { Two-site IRMA } \\ 2 & \text { A: Amerlex M RIA } \\ & \text { B: Amerlite CLA (luminescent 2-site) } \\ 3 & \text { Solid phase double antibody RIA (in house) } \\ & \text { (two variants) } \\ 5 & \text { DELFIA (IFMA) } \\ 6 & \text { A: Double antibody solid phase RIA (DPC) } \\ & \text { B: FSH IRMA (DPC) } \\ & \text { C:Two-site CEIA } \\ 7 & \text { MAIA-Clone (2-site IRMA) (Serono) } \\ 8 & \text { RIA } \\ 9 & \text { DELFIA } \\ 15 & \text { Omnia FSH IRMA (IDS) } \\ 17 & \text { In-house double-antibody RIA } \\ 19 & \text { Double antibody RIA (DPC) } \\ 20 & \text { MYRIA } \\ 21 & \text { Coated tube IRMA (FSHCTK-2 Sorin) } \\ 22 & \text { WHO matched reagent programme (1993) RIA } \\ 24 & \text { Two-site coated tube IRMA (EuroDiagnostica) } \\ 27 & \text { FEIA (Auraflex) } \\ 28 & \text { A: CLA } \\ 29 & \text { B: DELFIA } \\ 30 & \text { DELFIA } \\ \text { In house two-site ELISA (two formats) }\end{array}$

$\mathrm{RIA}=$ radioimmunoassay; $\mathrm{IRMA}=$ immunoradiometric assay; $\mathrm{FEIA}=$ fluorescent enzyme immunoassay; $\mathrm{CLA}=$ chemiluminescent assay ELISA = enzyme-linked immunoassay, $\mathrm{CEI} A=$ chemiluminescent enzyme immunoassay; IFMA = immunofluorometric assay.

Amerlex M, Amerlite, DELFIA, MAIA-Clone, Omnia, MYRIA, FSHCTK-2 and Auraflex are proprietary names for assays used.

DPC (Diagnostic Products Corporation) (Los Angeles, CA, USA);

Serono-Baker Diagnostics Inc. (Allentown, PA, USA); IDS (Immunodiagnostic Systems Ltd) (Boldon, UK); Sorin Biomedica (Saluggia, Italy);

EuroDiagnostica (Apeldoorn, The Netherlands); WHO matched reagent programme (see Sufi et al. 1991).

preparations have been published (De Boer \& Mannaerts 1990, Hard et al. 1990, Van Weezenbeek et al. 1990, Amoresano et al. 1996, Harris et al. 1996).

The bulk solutions were diluted in an appropriate buffer containing excipients and were distributed into ampoules as $1.0 \mathrm{ml}$ aliquots according to WHO guidelines. Ampoule contents for each preparation are summarised on Table 1 .

The second and third IS for urinary FSH and LH for bioassay (ISU) Ampoules (coded 71/223 and 71/264) contained a preparation of urinary FSH and $\mathrm{LH}$ with a defined content of 54 IU FSH and 46 IU LH per ampoule (Storring et al. 1976, WHO Expert Committee on Biological Standardization 1989, 1994). During the period of this study the stocks of the second ISU were effectively exhausted and the third ISU was established. These two materials appear to be indistinguishable, as previously indicated, and were used interchangeably throughout this study except where they have been directly compared with each other.
Participants and assays contributed to the study

The participants in this study are listed in Table 2 . Throughout this report each laboratory is identified by a number between 1 and 30 which does not relate to the order of listing. (NB Not all numbers have been used.)

\section{In vivo bioassays}

Ten laboratories contributed in vivo bioassays based on the gonadotrophin augmentation of ovarian weight gain. These assays are essentially similar and conform to relevant pharmacopoeial monographs: laboratories 25 and 30, European Pharmacopoeia (1986); laboratories 11, 18 and 23, British Pharmacopoeia (1988); laboratories 7, 16, 17, 22 and 29 (Steelman \& Pohley 1953). Each participant was requested to carry out at least two independent assays for each preparation tested preferably using at least three dose levels of each preparation so that linearity and parallelism of dose-response lines could be examined. For the purposes of this study an independent assay was considered to be one in which a freshly made up ampoule of test preparation was tested on a separate occasion using fresh batches of reagents where feasible. Participants were also asked to contribute full results of their assays including all raw data for analysis at NIBSC.

\section{In vitro bioassays and radioreceptor assays}

Nine laboratories contributed in vitro bioassays by six methods and three laboratories contributed radioreceptor assays by four methods. Laboratories 9, 10, 26, 28, 29 and 30 used Sertoli cells from immature rats (Guillou et al. 1986, Padmanabhan et al. 1987) and determined various end points including aromatase activity (laboratory 9), plasminogen activator (laboratory 26) and cAMP production. Four laboratories used human or rat $\mathrm{rFSH}$ receptor expressed in various cell types (Tilly et al. 1992, Gudermann et al. 1994): laboratory 7, mouse Y1 adrenal cells; laboratory 9, L cells; laboratory 12, human foetal kidney 293 cells; and laboratory 29 CHO cells. End points measured included progesterone and cAMP. Laboratory 8 used aromatase activity in rat granulosa cells (Miro et al. 1991). For the radioreceptor assays laboratories 9,10 and 29 used calf testis as a source of FSH receptor (Cheng 1975) and laboratory 29 additionally used human rFSH receptor expressed in $\mathrm{CHO}$ cells. Participants were asked to examine preparations using assay designs and contributing results as described for in vivo bioassays.

\section{Immunologically based assays}

Nineteen laboratories using 24 assay systems contributed immunologically based assays to the study (Table 3 ). Seven laboratories contributed RIA (defined as one-site assays for the purposes of analysis in this study), seven laboratories 
Table 4 Laboratory geometric mean estimates with 95\% fiducial limits obtained by in vivo bioassay for the FSH content of the IS for human urinary FSH (urofollitropin), in ampoules coded 92/512, of the IS for human $\mathrm{rFSH}$, in ampoules coded 92/642, and of a preparation of $\mathrm{rFSH}$ in ampoules coded 92/510, expressed as IU of the ISU per ampoule. The number of independent estimates contributing to each mean estimate is shown in parentheses. Estimates are weighted geometric means of homogeneous estimates, except as marked* when estimates are the unweighted geometric means of heterogeneous estimates. NA denotes that the preparation was not assayed. The estimates in braces result from deletion of the single estimate with the largest contribution to the heterogeneity chi-square value for all estimates

\begin{tabular}{|c|c|c|c|}
\hline & $92 / 512$ & $92 / 642$ & $92 / 510$ \\
\hline \multicolumn{4}{|c|}{ Laboratory number } \\
\hline 7 & 97 (1) 70-143 & $133 *(2) 119-148$ & $97 *$ (2) $20-467$ \\
\hline 11 & 112 (5) 98-127 & 139 (5) 120-161 & 91 (5) 78-108 \\
\hline 16 & 147 (2) 119-181 & 138 (4) 118-161 & 100 (3) 86-115 \\
\hline 17 & NA & 124 (2) 101-152 & 90 (2) 76-106 \\
\hline 22 & 129 (1) 104-160 & 144 (1) 116-182 & 73 (1) 58-97 \\
\hline 23 & $\begin{array}{l}151 \text { (2) } 129-177 \\
{[146(1) 120-179]}\end{array}$ & 160 (2) 130-195 & 98 (2) 84-114 \\
\hline 25 & 120 (2) 102-140 & 145 (2) 119-176 & 101 (2) 83-124 \\
\hline 29 & 125 (2) 104-150 & 156 (2) 128-189 & 102 (2) 86-121 \\
\hline 30 & 102 (3) 85-123 & 132 (5) 117-148 & 105 (4) 67-165 \\
\hline All estimates & $\begin{array}{l}126^{*}(18) \text { 110-142 } \\
{[121 \text { (17) } 113-128]}\end{array}$ & 138 (25) 131-145 & 95 (23) 90-100 \\
\hline
\end{tabular}

contributed IRMA and eight laboratories contributed other assay types including ELISA and similar assays with different detection modes including chemiluminescence and fluorescence (defined as two-site assays for the purposes of analysis in this study). The majority of these assays were commercially available assays in kit form which included a kit standard calibrated against the International Reference Preparation for pituitary FSH (Code 78/549). Other assays were in-house assays with standards derived from various sources including the National Institutes for Diabetic, Digestive and Kidney Diseases (NIDDK, Bethesda, MD, USA). Participants were asked to carry out at least two independent assays, each to include each of the preparations supplied at as many dilutions as practicable (at least three and preferably six or more) to provide information about the dose-response curves and to return full results of their assays for analysis at NIBSC.

\section{Statistical analysis}

In vivo bioassays The majority of participants contributed data for both body weight and organ weight for each response. These data were examined using both organ weight only and organ weight relative to body weight, both as given and following $\log$ transformation as the assay response. Within each laboratory the response used for subsequent analysis was that which best satisfied the criteria of statistically valid assays with homogeneous groups of responses and precise estimates of relative potency. Doses with mean responses which contributed significantly to non-linearity and which appeared to be at the extremes of the response range were omitted from analysis.

Log potency estimates for the $\mathrm{rFSH}$ and purified urinary FSH in terms of the ISU were obtained as the displacement of parallel $\log$ dose- $(\log )$ response lines from analysis as paired or multiple parallel-line assays. Statistical weights for the paired analysis were computed as the reciprocal of the variance of the $\log$ potency. Assays described as statistically valid showed no significant $(P>0 \cdot 05)$ deviations from linearity or parallelism.

In vitro bioassays, receptor assays and immunoassays For each assay response data (as far as possible the directly measured values) were plotted against log dose and assessed both graphically and statistically for outliers, consistency of increase (or decrease) of dose-response relationship and homogeneity of variances. Groups of responses with an excessively large variance relative to the variances of other groups in the same assay were examined further. If such groups consisted of only two replicates, both were deleted; individual outlying responses in groups of three or more replicates were deleted. Response-error relationships were examined and where variances showed a significant increase with increasing response, the relationship of variance to mean response raised to a positive power, $\alpha$, was fitted using a maximum likelihood method (Raab 1981). This relationship, with $\alpha$ taken as the average value of $\alpha$ for assays of the same type from the same laboratory, was used in subsequent analysis. Dose-response relationships were assessed for conformity to a four parameter 


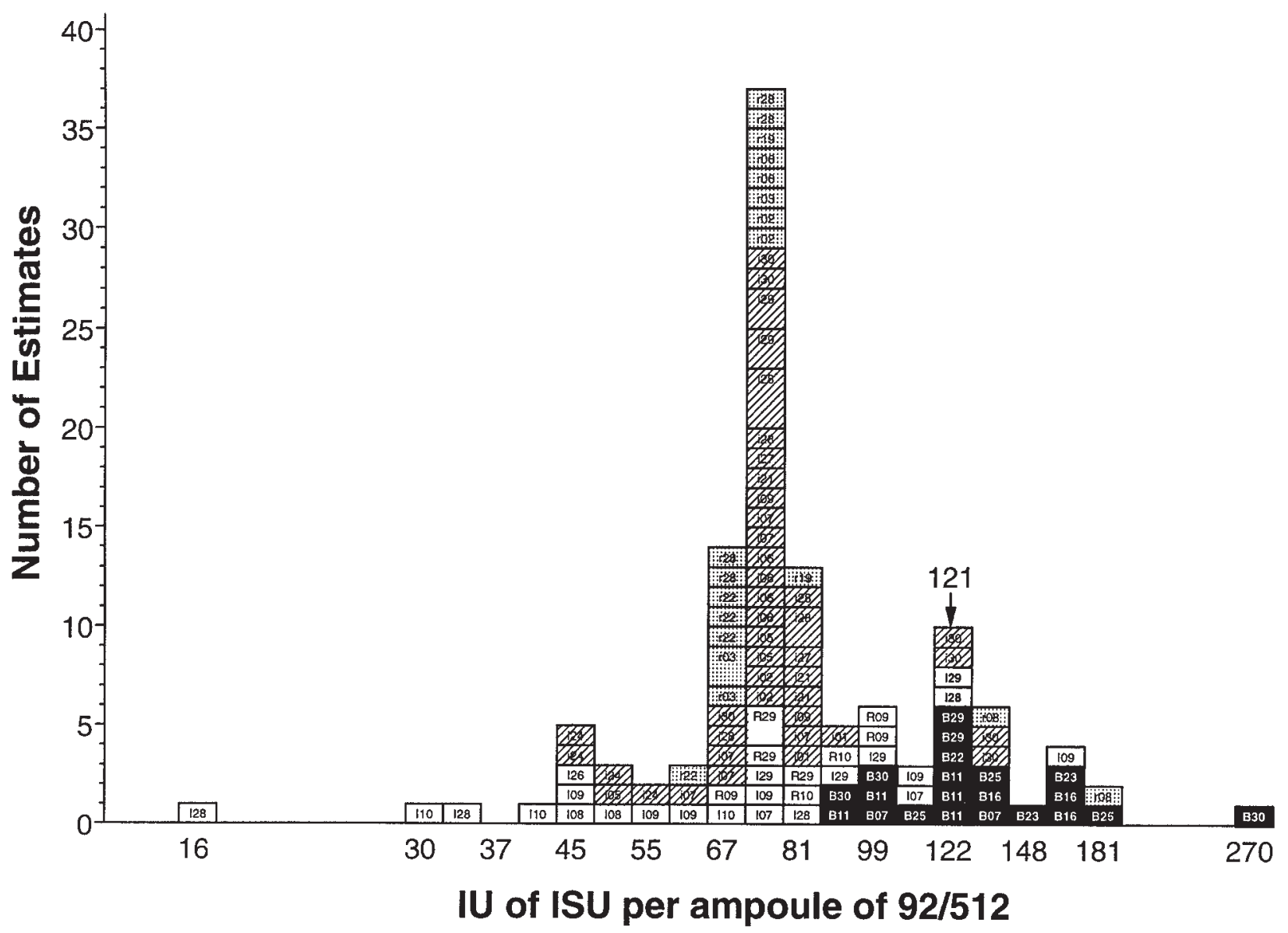

Figure 1 Individual estimates of FSH content for ampoules of 92/512, expressed as IU of the ISU in ampoules coded 71/223 plotted on a $\log$ scale. Each square denotes an individual estimate. The three character code in the square indicates the assay type (B denotes in vivo assay; $R$ receptor assay, I in vitro assay; i two-site immunoassay and $r$ one-site immunoassay) followed by the laboratory number. Shading has been added to emphasise assay type: open squares are in vitro bioassays or receptor assays, solid squares are in vivo assays, hatched squares are one-site assays and dotted squares are two-site assays. Double squares with a single code indicate two estimates which were obtained in the same assay using differently labelled ampoules. The arrowed number shows the weighted geometric mean of the estimates by bioassay.

logistic function, maximum and minimum responses for each assay were determined using this model, and data for each assay were analysed as parallel-line assays using a logit transformation of the responses (and the program WRANL (Gaines-Das \& Tydeman 1982)). Log dose-logit response lines were assessed for linearity and parallelism and $\log$ potency estimates were obtained as the displacement of parallel log dose-logit response lines. In a few cases either the response range was so limited that a four parameter logistic model could not be assessed or conformity to this model was not satisfactory and the responses from the linear part of the response range have been analysed as described for in vivo bioassays. Slopes of $\log$ dose-logit response lines were examined for any consistent trends. Differences between slopes of coded duplicate preparations were used to provide a direct measure of intra-assay slope variability.
Combination of potency estimates Estimates of log potency from analysis of in vivo bioassays for specified pairs of preparations were examined for heterogeneity using a chi-squared test and combined as weighted geometric means where estimates were homogeneous, or unweighted geometric means where estimates were heterogeneous. Estimates of log potency from analysis of in vitro bioassays, receptor assays and immunoassays were combined as unweighted geometric means. Fiducial limits for unweighted means were determined from the variance of the logarithms of the combined estimates. Comparisons among groups of estimates were made using unweighted analysis of variance of the $\log$ potency estimates of the groups compared.

Inclusion, where possible, of a coded duplicate of one of the FSH preparations provided a direct measure of the intra-assay variation of potency estimates calculated as the 


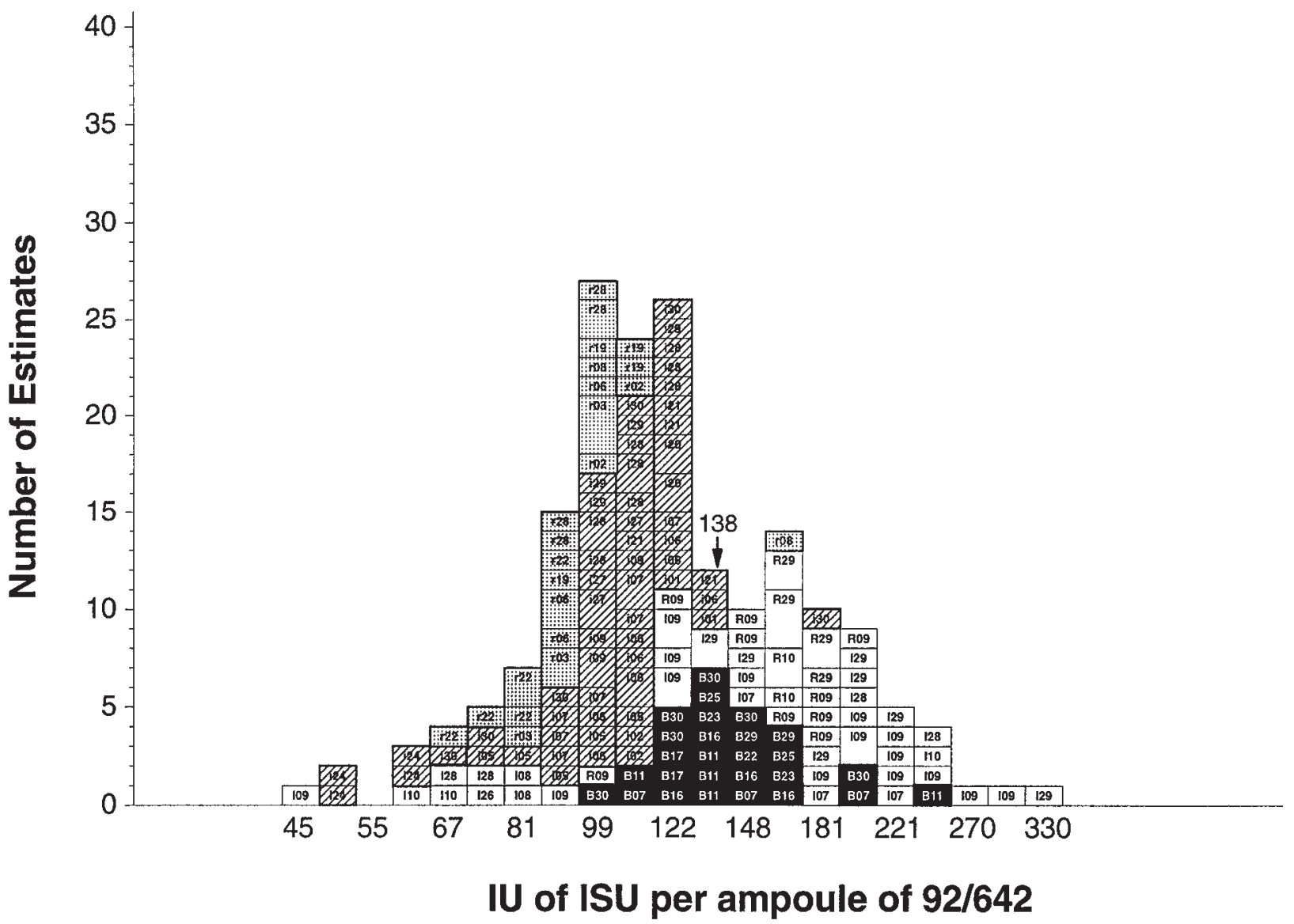

Figure 2 Individual estimates of the FSH content for ampoules coded 92/642, expressed as IU of the ISU per ampoule. Codes and shading in squares as for Fig. 1. The arrowed number shows the weighted geometric mean of the estimates by bioassay.

deviation of the $\log$ relative potency estimate of the duplicate in terms of the identical material from its expected value of 0 . This is taken to provide a measure of the minimum inherent variability in these systems, and is taken as the base against which comparisons among other estimates are considered.

\section{Results}

Comparison of the second and third ISU

Over the period of this study the second ISU (in ampoules coded 71/223) was replaced by the third ISU (in ampoules 71/264). These two preparations were derived from the same bulk material and were ampouled under identical conditions. Several of the laboratories received ampoules of both preparations. Pairwise comparison of these two preparations confirmed the continuity of this unitage. Nine independent in vivo bioassays in six laboratories were statistically valid and gave estimates which were homogeneous with a weighted geometric mean (95\% fiducial limits) of $0.99(0.91-1 \cdot 08)$ IU of the second ISU equivalent in activity to 1 IU of the third ISU (data not shown). These two preparations were also compared in 39 assays in 14 immunoassay systems. Slopes of log dose-logit response lines for these two preparations were similar and estimates of the relative potency of $71 / 264$ in terms of $71 / 223$ (IU/IU) ranged from 0.80 to 1.20 with an unweighted geometric mean (95\% limits) of $1.02(0.99$ to 1.05$)$ IU/IU. For subsequent analysis these two preparations have been treated as identical and referred to as a single preparation (ISU). Where one (but not both) of the second or the third ISU, was included in an assay, it has been recorded as the ISU. Where both preparations were included in the same assay, responses have been combined and treated as if given by a single preparation so that maximum precision is obtained for calibrations in terms of the ISU.

\section{Dose-response relationships for $r F S H$, purified urinary FSH} and ISU

In vivo bioassays Seven of the ten participants provided data for both body weights and organ weights. In general 


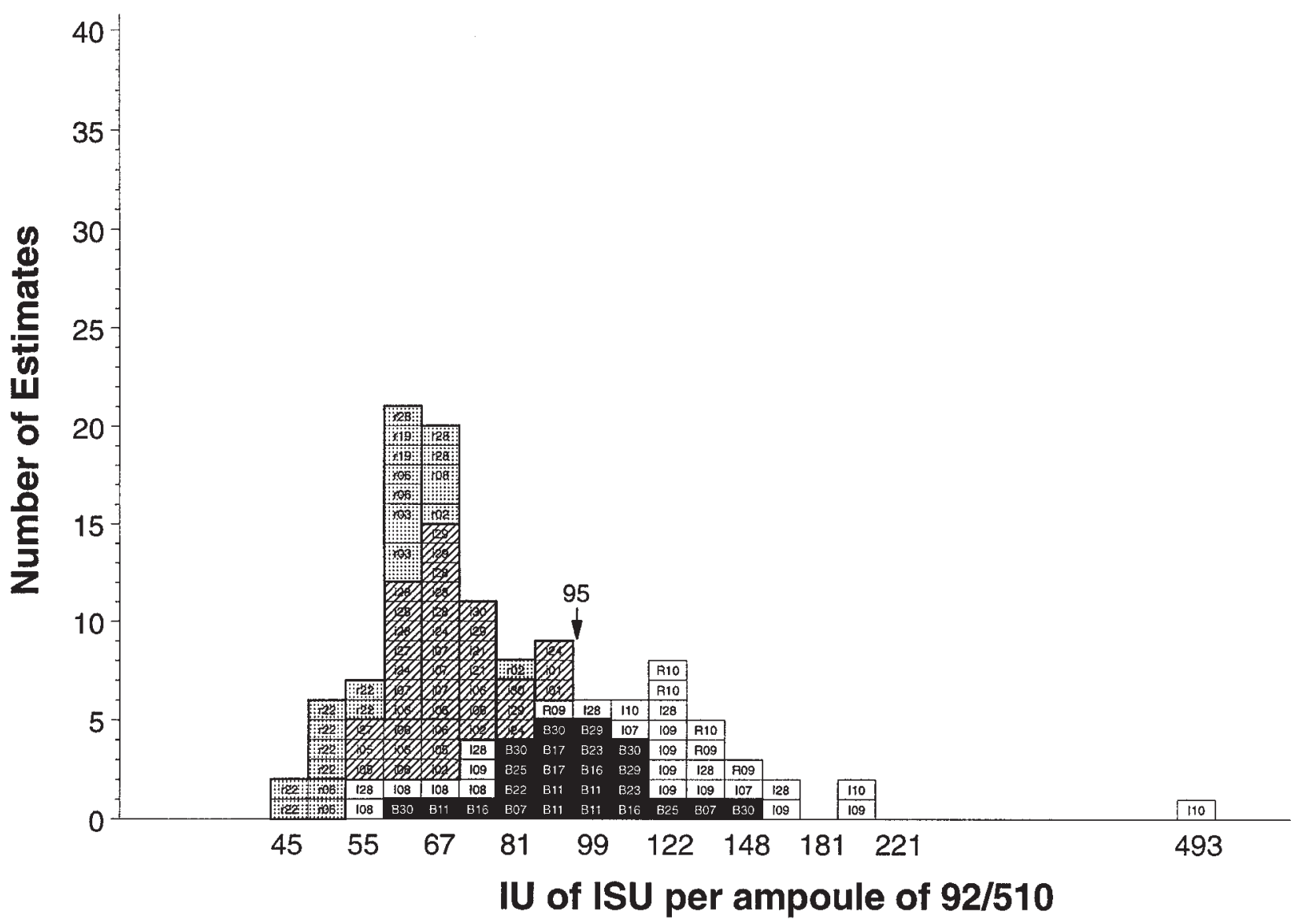

Figure 3 Individual estimates of the FSH content for ampoules coded 92/510, expressed as IU of the ISU per ampoule. Codes and shading in squares as for Fig. 1. The arrowed number shows the weighted geometric mean of the estimates by bioassay.

the response used for analysis was log transformed organ weight relative to body weight. This response was also used for consistency in the few cases where there was no clear advantage of one response transformation, and for the one participant who supplied this response without separate data for body weights. Two participants supplied organ weight responses only; in one case log transformed response, and in the other untransformed response, gave more precise estimates of relative potency. Analysis of pairwise comparisons was statistically valid for the majority of assays with fewer than $5 \%$ exceptions.

In vitro bioassays and receptor assays Log dose-logit response lines for the four preparations in these assays did not show consistent significant deviations from linearity or parallelism; except in the receptor assays using $\mathrm{CHO}$ cells there was a tendency for $92 / 642$ to give steeper doseresponse lines than the ISU and 92/512 (92/510 was not included in these assays). However, only marked instances of non-parallelism would have been detected for many of these assays because of the limited number of doses tested in a number of assays and because of the variability of the measured responses in many assays.

Immunoassays For 16 of the 24 assay systems in this study $\log$ dose-logit response lines did not differ significantly and consistently from linearity or parallelism, although all four preparations were not included in five of those assay systems. In laboratory 24 , these four preparations were tested at a single dose and results from this assay system have been excluded from this analysis. In the remaining assay systems there was a tendency for the dose-response lines for the ISU or $92 / 642$, or rarely both, to differ consistently from those of other preparations. Although these data indicate that some differences in sensitivity or selectivity exist between different assay systems, which is to be expected, the magnitude of the differences are not sufficient to preclude use of any preparation as an IS.

\section{Variability of estimates}

A measure of the within-assay accuracy and precision of the assays contributed was enabled by inclusion within 
Table 5 Unweighted laboratory geometric mean estimates (gms), with 95\% fiducial limits based on the variance of the logarithms of the means combined, for the FSH content of the IS for human urinary FSH (urofollitropin), in ampoules coded 92/512, of the IS for human $\mathrm{rFSH}$, in ampoules coded 92/642, and of a preparation of $\mathrm{rFSH}$ in ampoules coded $92 / 510$ obtained by in vitro bioassays and receptor assays, expressed as IU of the ISU per ampoule. The number of independent estimates contributing to each mean estimate is shown in parentheses. NA denotes that the preparation was not assayed

\begin{tabular}{|c|c|c|c|}
\hline & $92 / 512$ & $92 / 642$ & $92 / 510$ \\
\hline \multicolumn{4}{|l|}{$\begin{array}{l}\text { Laboratory number } \\
\text { In vitro bioassays - Sertoli cells }\end{array}$} \\
\hline 9 & 84 (4) 33-213 & 182 (4) $121-274$ & 145 (4) $93-226$ \\
\hline 10 & 44 (3) $15-130$ & 103 (3) 15-708 & 220 (3) 36-1345 \\
\hline 26 & $43(1)$ & $77(1)$ & NA \\
\hline 28 & 48 (4) 12-194 & 124 (4) 43-352 & 102 (4) 58-179 \\
\hline 29 & 96 (2) $70-132$ & 170 (2) $16-1760$ & NA \\
\hline \multicolumn{4}{|l|}{ In vitro bioassays - various cells } \\
\hline $7(\mathrm{Y} 1)$ & 89 (2) 10-790 & 173 (2) 28-1070 & 123 (2) 16-926 \\
\hline 8 (Granulosa) & 46 (2) $26-82$ & 77 (2) $73-80$ & 62 (2) 29-134 \\
\hline $9(\mathrm{~L})$ & 64 (2) 14-293 & 135 (2) 18-1030 & 120 (2) 10-1480 \\
\hline $29(\mathrm{CHO})$ & 96 (2) 6-1570 & 203 (2) 14-3040 & NA \\
\hline Geometric means of laboratory gms & 64 (9) 49-84 & 131 (9) 98-173 & 120 (6) 78-186 \\
\hline \multicolumn{4}{|l|}{ Receptor assays - calf } \\
\hline 9 & 83 (3) 53-131 & 154 (3) 105-224 & 120 (3) 60-239 \\
\hline 10 & 83 (2) 46-149 & 166 (2) 135-205 & 123 (2) 94-160 \\
\hline 29 & 75 (2) 55-104 & 168 (2) 146-193 & NA \\
\hline \multicolumn{4}{|l|}{ Receptor assays - $\mathrm{CHO}$} \\
\hline 29 & 79 (2) 52-118 & 180 (2) 145-223 & NA \\
\hline Geometric mean of laboratory gms & 80 (4) 74-86 & 167 (4) 150-185 & 121 (2) 107-138 \\
\hline
\end{tabular}

some in vitro and receptor assays and within most immunoassays of coded duplicates (ampoules of the same material coded with different letters for assay) and by the need to confirm the continuity of unitage between the second and third ISU. It is expected that the relative potency of coded duplicates to one another should be 1 , and similarly of the third and second ISU assuming these preparations to be identical. Deviations of the relative potency from the value of 1 reflect variability within the assay. Consistent differences within a laboratory from the value of 1 and consequent differences between laboratories may result if there is a consistent bias in the assay system.

Comparison by in vivo bioassay and by immunoassay of the third ISU with the second ISU gave estimates of relative potency which did not differ significantly from 1 and which showed no greater variability between laboratories and assays than within laboratories and assays. Comparison by immunoassay gave geometric coefficients of variation $(\mathrm{gcv})$ of about $10 \%$ both within and between assays and laboratories.

Coded duplicates of one of the rFSH preparations or of the purified urinary FSH were included in most immunoassays and a number of in vitro or receptor assays. For each pair of duplicates and each type of assay, estimates showed within- and between-assay variabilities and betweenlaboratory variabilities which were broadly similar. All estimates for duplicates were therefore used to give pooled variances for each assay type. The $\mathrm{gcv}$ for immunoassays was about $10 \%$ or less both within and between assays and was similar or marginally larger between laboratories. For in vitro bioassays the within- and between-assay gcvs were each about 50\% while laboratory geometric means had a gcv of about $30 \%$. For receptor assays, variances within and between assays and between laboratories were similar, with gcvs generally between 10 and 15\%.

Variability between assays within laboratories was examined directly for all comparisons by each type of assay, including in vivo bioassays. For immunoassays the intralaboratory variability was smallest with gcv less than $10 \%$ for virtually all comparisons. For receptor assays the intra-laboratory gcv was generally between 10 and $15 \%$. For in vivo bioassays the intra-laboratory gcv, determined directly using unweighted estimates of potency, was generally about $25 \%$. In vitro bioassays gave the largest intra-laboratory variability with gcvs from 40 to $70 \%$.

Calibration of the IS for FSH, human urinary (urofollitropin) in ampoules coded 92/512, the IS for human $r F S H$ in ampoules coded $92 / 642$ and a preparation of $r F S H$ in ampoules coded 92/510 in terms of the ISU

Calibration by in vivo bioassays Laboratory geometric mean estimates are given in Table 4 and individual estimates are shown in Figs 1-3. 
Table 6 Unweighted laboratory geometric mean estimates (gms) for the FSH content of the IS for purified urinary FSH in ampoules coded 92/512, of the IS for $\mathrm{rFSH}$ in ampoules coded 92/642, and of a preparation of rFSH in ampoules coded 92/510 obtained by immunoassay, expressed as IU of the ISU per ampoule. The number of independent estimates contributing to each mean estimate is shown in parentheses beside the laboratory code where there were the same number of estimates for each preparation, and otherwise beside the mean estimate. The 95\% fiducial limits for the unweighted geometric mean of laboratory mean estimates is based on the variance of the logarithms of the estimates combined. NA denotes not assayed

\begin{tabular}{|c|c|c|c|}
\hline & $92 / 512$ & $92 / 642$ & $92 / 510$ \\
\hline \multicolumn{4}{|l|}{ Laboratory number } \\
\hline \multicolumn{4}{|l|}{ Two-site immunoassays } \\
\hline $1(2)$ & 86 & 134 & 88 \\
\hline $2(2)$ & 75 & 110 & 72 \\
\hline $5(3)$ & 65 & 93 & 61 \\
\hline $6 \mathrm{~A}(2)$ & 74 & 122 & 65 \\
\hline $6 \mathrm{~B}(2)$ & 75 & 108 & 65 \\
\hline $7(4)$ & 70 & 107 & 65 \\
\hline $9(2)$ & 77 & 103 & NA \\
\hline $20(2)$ & NA & 122 & NA \\
\hline $21(2)$ & 78 & 126 & 73 \\
\hline $27(2)$ & 77 & 110 & 65 \\
\hline $28 \mathrm{~A}(3)$ & 78 & 111 & 65 \\
\hline 28B (3) & 76 & 115 & 67 \\
\hline $29 A(2)$ & 72 & 101 & 66 \\
\hline $29 B(2)$ & 74 & 116 & 78 \\
\hline $30 \mathrm{~A}(2)$ & $131 *$ & NA & NA \\
\hline $30 \mathrm{~B}$ & $85(5)^{*}$ & $97(6)^{*}$ & $73(2)^{*}$ \\
\hline Geometric mean of laboratory gms & 78 (15) $72-86$ & 111 (15) 105-117 & 69 (13) 65-73 \\
\hline Omitting estimates marked* & 75 (13) $72-78$ & 112 (14) 106-119 & 69 (12) 65-73 \\
\hline \multicolumn{4}{|l|}{ One-site immunoassays } \\
\hline $2(2)$ & 73 & 105 & 74 \\
\hline $3(2)$ & 67 & 91 & 60 \\
\hline $6(2)$ & 72 & 95 & 55 \\
\hline $8(1)$ & $144^{*}$ & $120^{*}$ & $66^{*}$ \\
\hline $19(2)$ & 78 & 101 & 61 \\
\hline $22(4)$ & 63 & 85 & 52 \\
\hline $28(3)$ & 72 & 96 & 65 \\
\hline Geometric mean of laboratory gms & 78 (7) 60-101 & 98 (7) 89-109 & 62 (7) 55-69 \\
\hline Omitting estimates marked* & $71(6) 65-77$ & 95 (6) 88-103 & 61 (6) 53-69 \\
\hline \multicolumn{4}{|l|}{ One-site and two-site immunoassays } \\
\hline Geometric mean of laboratory gms & 78 (22) $72-86$ & 107 (22) 101-113 & 66 (20) 63-70 \\
\hline Omitting estimates marked* & 74 (19) $71-76$ & 107 (20) 101-113 & 66 (18) 62-70 \\
\hline
\end{tabular}

Comparison of the IS for human urinary FSH (urofollitropin), with the ISU was carried out in 21 independent assays in eight laboratories. After deletion of estimates from two statistically invalid assays and of one estimate which contributed significantly to the heterogeneity chi-squared value, the remaining estimates were broadly similar $(P \sim 0.03$ for chi-squared value for heterogeneity) with an unweighted geometric mean of 126 IU of ISU per ampoule. Deletion of an additional estimate which contributed most to the heterogeneity chi-squared value gave a group of 17 estimates which did not differ significantly from homogeneity and which had a weighted geometric mean (95\% fiducial limits) of 121 (113-128) IU of the ISU per ampoule of $92 / 512$.
Comparison of the IS for human rFSH with the ISU was carried out in 26 independent assays in nine laboratories. The weighted geometric mean $(95 \%$ fiducial limit) of 25 homogeneous estimates (after deletion of one estimate from a statistically invalid assay) was 138 (131-145) IU of the ISU per ampoule of 92/642.

Comparison of the $\mathrm{rFSH}$ preparation in ampoules coded $92 / 510$ with the ISU was carried out in 24 independent assays in nine laboratories. All assays were statistically valid, but one estimate contributed significantly to the heterogeneity chi-squared value both within the laboratory in which it was obtained, and over all estimates, and has been deleted. The remaining 23 estimates did not differ significantly from homogeneity and gave a weighted geometric 
Table 7 Laboratory geometric mean estimates with 95\% fiducial limits for the FSH content of the IS for rFSH in ampoules coded 92/642, and of a preparation of $\mathrm{rFSH}$ in ampoules coded 92/510 obtained by in vivo bioassay, expressed as IU of the IS for purified urinary FSH per ampoule, and for the FSH content of a preparation of rFSH in ampoules coded 92/510 obtained by in vivo bioassay, expressed as IU of the IS rFSH per ampoule. The number of independent estimates contributing to each mean estimate is shown in parentheses. Estimates are weighted geometric means of homogeneous estimates, except as marked* when estimates are the unweighted geometric means of heterogeneous estimates

92/642 (as IU of 92/512)

Laboratory number
7
11
16
17
18
22
23
25
29
30

All estimates

\begin{tabular}{l}
\hline 157 (2) $134-184$ \\
148 (3) $125-177$ \\
113 (3) $97-133$ \\
NA \\
NA \\
137 (1) $111-170$ \\
122 (2) $99-149$ \\
145 (1) $111-192$ \\
155 (2) $128-189$ \\
NA
\end{tabular}

138 (14) $129-148$ $\mathbf{9 2 / 5 1 0}$ (as IU of 92/512)

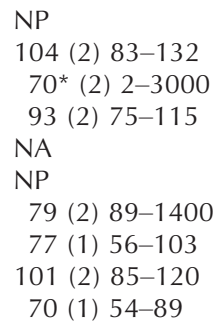

NP

104 (2) 83-132

70 * (2) $2-3000$

93 (2) $75-115$

NA

NP

79 (2) $89-1400$

77 (1) $56-103$

101 (2) $85-120$

70 (1) 54-89

92/510 (as IU of 92/642)

85 (12) 79-92

NP, Log dose-response lines were non-parallel.

mean (95\% fiducial limits) of $94 \cdot 8(90 \cdot 2-99 \cdot 6)$ IU of ISU per ampoule of $92 / 510$.

Calibration by in vitro bioassays and receptor assays Laboratory geometric mean estimates are given in Table 5 and individual estimates are shown in Figs 1-3.

Estimates by receptor assays for $92 / 512$, with an unweighted geometric mean of laboratory means (95\% limits) of 80 (74 to 86 ) IU per ampoule, were markedly smaller than those obtained by in vivo bioassay. For both of the rFSH preparations 92/642 and 92/510, estimates by receptor assays were significantly larger than those obtained by in vivo bioassays, namely 167 (150-185) IU per ampoule for 92/642 and 121 (107 to 138) IU per ampoule for $92 / 510$. Estimates by in vitro bioassays varied widely with overall means similar to those obtained by receptor assays. Because of the large intra-laboratory variability, the extent to which the estimates by in vitro bioassay in the different laboratories reflect inter-laboratory differences is not clear. For each calibration, the inter-laboratory variability for estimates by in vitro bioassay tends to be larger than the pooled intra-laboratory variability, with the largest for the calibration of $\mathrm{rFSH}$ preparation coded $92 / 510$.

Calibration by immunoassays Laboratory mean geometric estimates are given in Table 6 and individual estimates are shown in Figs 1-3. For each calibration, estimates by immunoassay were significantly smaller than estimates by in vivo bioassay with overall estimates (95\% limits) for $92 / 512,92 / 642$ and $92 / 510$ of 74 (71 to 76$)$, 107 (101 to 113 ) and 66 (62 to 70) IU of ISU per ampoule. The ratio of in vivo bioactivity to the overall mean immunoreactivity was 1.7 for $92 / 512,1.29$ for 92/642 and 1.44 for $92 / 510$.

For calibration of each of the three ampouled preparations, over all immunoassays or over one- and two-site immunoassays separately, the inter-laboratory variability was larger than the pooled intra-laboratory variability. For two-site immunoassays and for all immunoassays considered together, the inter-laboratory variability for calibration of $92 / 512$ was less than half that for calibration of 92/510 or 92/642. Estimates by one-site assays were less, for each calibration, than estimates by two-site assays with the difference being least for calibration of 92/512. (One-site estimates were 5, 15 and 12\% less than estimates from two-site assays for 92/512, 92/642 and 92/510 respectively.)

Comparison of the IS for human rFSH in ampoules coded $92 / 642$ and a preparation of human rFSH in ampoules coded $92 / 510$ with the IS for human urinary FSH (urofollitropin) coded $92 / 512$

Comparisons by in vivo bioassay The IS for human rFSH was compared directly with the IS for FSH, human, urinary in 15 in vivo bioassays (Table 7 and Fig. 4). One of these estimates contributed significantly to the heterogeneity chi-squared value both within the laboratory in which it was obtained, and over all estimates, and has been deleted. The remaining 14 estimates did not differ significantly from homogeneity and had a weighted geometric mean (95\% limits) of 138 (129-148) IU of the IS for FSH, human urinary per ampoule. This value is virtually identical to the value obtained by calibration of the IS for human $\mathrm{rFSH}$ in terms of the ISU. 


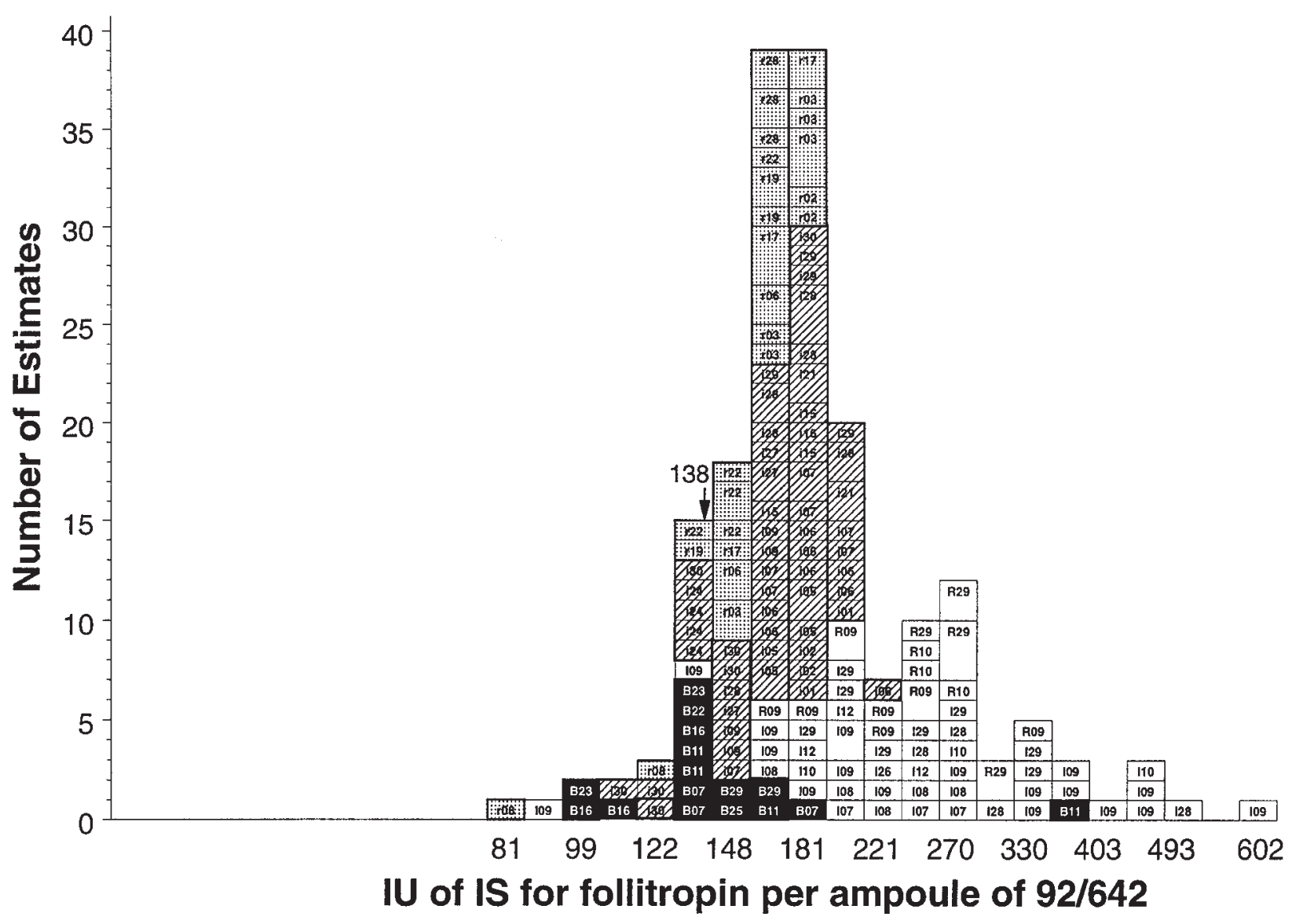

Figure 4 Individual estimates of the FSH content for ampoules coded 92/642 expressed as IU of the IS for urinary FSH (in ampoules coded 92/512) per ampoule of 92/642. Codes and shading are as in Fig. 1. The arrowed number shows the weighted geometric mean of the estimates by bioassay.

The $\mathrm{rFSH}$ in ampoules coded $92 / 510$ was compared directly with the IS for human urinary FSH in 16 in vivo bioassays (Table 7 and Fig. 5). Three of these assays showed significant deviations from parallelism and were deleted. Further deletion of one estimate, which contributed most greatly to the heterogeneity chi-squared value, gave a group of estimates which did not differ significantly from homogeneity, and which had a weighted geometric mean (95\% limits) of 85 (79 to 92) IU of the IS for human urinary FSH per ampoule. This is similar to the value obtained by calibration of this preparation in terms of the ISU, although being based on only half the number of assays, may be less reliable.

Comparisons by in vitro bioassays and receptor assays Estimates obtained by direct comparisons of 92/ 642 and 92/510 with the IS for human urinary FSH are shown in Table 8 and Figs 4 and 5. Overall estimates by both in vitro bioassays and receptor assays are some two times larger than those obtained by in vivo bioassay using the IS for human urinary FSH as standard. These estimates are also larger, by a factor of about $1 \cdot 5$, than estimates obtained by calibration of these preparations by in vitro bioassays or receptor assays in terms of the ISU. The inter-laboratory variability for comparisons by in vitro bioassay of $92 / 642$ with the IS for human urinary FSH is not larger than the intra-laboratory variability. However, comparisons of 92/510 with the IS for human urinary FSH show somewhat less inter-laboratory consistency. There are receptor assays in only two laboratories for the latter comparison. However, comparison of $92 / 642$ with the IS for human urinary FSH gave a larger estimate in receptor assays using $\mathrm{CHO}$ cells expressing $\mathrm{rFSH}$ receptors than in assays using the classical calf testis membrane assay.

Comparisons by immunoassays Estimates obtained by direct comparisons of $92 / 642$ and $92 / 510$ with the IS for human urinary FSH are shown in Table 9 and Figs 4 and 5 . These estimates are significantly larger than those obtained by immunoassay using the ISU as standard and are also larger than estimates obtained by in vivo bioassay for these comparisons. Excluding laboratories 30 


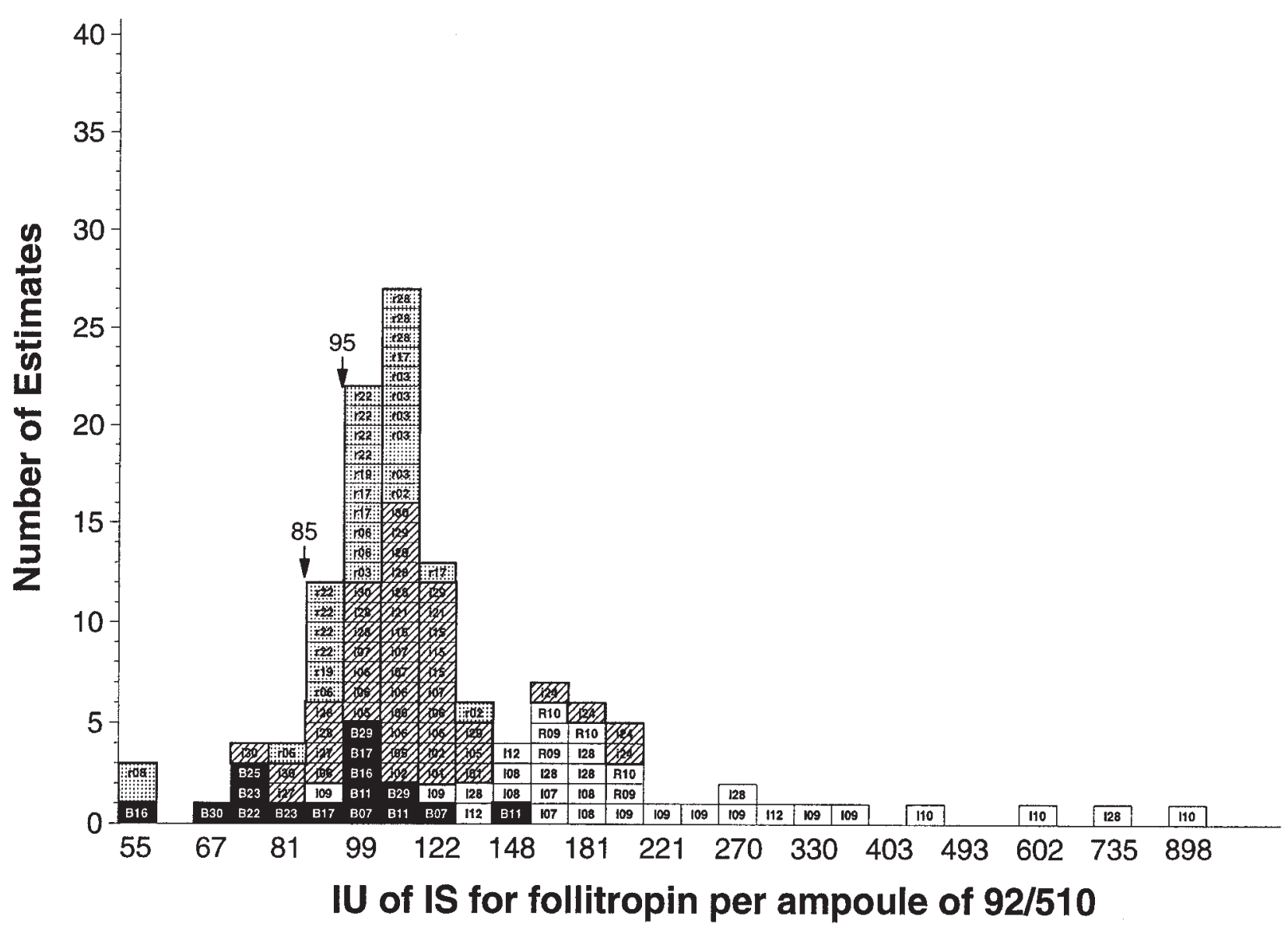

Figure 5 Individual estimates of the FSH content for ampoules coded 92/510 expressed as IU of the IS for urinary FSH (in ampoules coded 92/512) per ampoule of 92/510. Codes and shading as in Fig. 1. The arrowed numbers show the weighted geometric mean of estimates by in vivo bioassay for 92/510 as IU of 92/512 (85) and, for comparison, 92/510 as IU of the ISU (95).

and 8 , the inter-laboratory variability for either comparison is slightly more variable than the intra-laboratory variability.

Comparison of a preparation of human $r F S H$ in ampoules coded $92 / 510$ with the IS for human $r F S H$

Comparisons by in vivo bioassay The $\mathrm{rFSH}$ preparation in ampoules coded 92/510 was compared directly with the IS for human rFSH in 22 in vivo bioassays (Table 7 and Fig. 6). Two of these assays showed significant deviations from parallelism and were deleted. The remaining 20 estimates did not differ significantly from homogeneity and had a weighted geometric mean (95\% limits) of 95 (89-100) IU of the IS for human $\mathrm{rFSH}$ per ampoule. This is virtually identical to the value obtained by calibration of this preparation in terms of the ISU and similar to the value obtained by comparison of this preparation with the IS for human urinary FSH.
Comparisons by in vitro bioassays and receptor assays Estimates obtained by direct comparisons of 92/ 510 with the IS for human $\mathrm{rFSH}$ are shown in Table 8 and Fig. 6. Overall estimates by both in vitro bioassays and receptor assays are larger than those obtained by in vivo bioassay using the IS for human $\mathrm{rFSH}$ as standard. However, these estimates are similar to estimates obtained by calibration of this preparation by in vitro bioassays or receptor assays in terms of the IS for human urinary FSH. The inter-laboratory variability for this comparison was not significantly larger than the intra-laboratory variability.

Comparisons by immunoassay Estimates obtained by direct comparisons of $92 / 510$ with the IS for human $\mathrm{rFSH}$ are shown in Table 9 and Fig. 6 . These estimates are about $10 \%$ smaller than those obtained by in vivo bioassay using the IS for human rFSH as standard, and about 30\% larger than those obtained by immunoassay using the IS for human urinary FSH as standard. With the previously 
Table 8 Unweighted laboratory geometric mean estimates (gms) with 95\% fiducial limits obtained by in vitro bioassays and receptor assays for the FSH content of the IS for rFSH in ampoules coded 92/642, and of a preparation of rFSH in ampoules coded 92/510, expressed as IU of the IS for purified urinary FSH per ampoule, and for the FSH content of a preparation of rFSH in ampoules coded 92/510, expressed as IU of the IS for human rFSH per ampoule. The number of independent estimates contributing to each mean estimate is shown in parentheses

Laboratory number
In vitro bioassays - Sertoli cells
9
10
26
28
29

In vitro bioassays - various cells

$7(\mathrm{Y} 1)$

8 (Granulosa)

9 (L)

12 (Human)

$29(\mathrm{CHO})$

Geometric mean of laboratory gms

Receptor assays - calf

9

10

29

Receptor assays - $\mathrm{CHO}$

29

Geometric mean of laboratory gms $\mathbf{9 2 / 6 4 2}$ (as IU of 92/512)

310 (4) 191-504

214 (2) 29-1600

235 (2) 163-338

209 (5) 166-263

255 (2) 153-425

206 (3) 142-299

256 (2) 234-279

243 (10) 220-268

224 (3) $199-251$
243 (2) $166-355$
270 (2) $170-427$

277 (2) 228-338

253 (4) 216-296
92/510 (as IU of 92/512)

209 (4) $84-521$

607 (3) 295-1250

NA

256 (4) 85-777

NA

92/510 (as IU of 92/642)

110 (4) 78-156

208 (6) 91-473

NA

114 (4) 58-223

NA

167 (2) 142-198

163 (2) 134-197

226 (2) 4-10 000

184 (3) 65-525

NA

233 (7) 153-354

98 (2) 81-120

111 (2) 49-251

122 (2) 2-10 000

123 (3) 62-246

NA

123 (7) 99-154

175 (3) 137-223

179 (2) 76-422

NA

108 (3) 79-148

100 (3) 89-113

NA

NA

177 (2) 151-208
NA

104 (2) 64-169 noted exclusions, the inter-laboratory variability for the comparison by immunoassay of $92 / 510$ and the IS for human $\mathrm{rFSH}$ was only marginally more variable than the inter-laboratory variability and similar to that obtained for the comparisons of the IS for human $\mathrm{rFSH}$ and the IS for human urinary FSH.

\section{Accelerated thermal degradation studies of the candidate standards}

Samples of each of the ampouled purified preparations of FSH which had been stored at elevated temperatures for 1-2 years (ATD samples) were compared within the same assay with ampoules of the same preparation which had been stored continuously at $-20{ }^{\circ} \mathrm{C}$. The ATD samples of $92 / 642$ did not differ significantly from the samples stored at $-20{ }^{\circ} \mathrm{C}$ in two in vivo bioassays. Estimates of the activity of ATD samples of both $92 / 510$ and 92/512 relative to samples stored continuously at $-20{ }^{\circ} \mathrm{C}$ in two to four bioassays showed a loss of about $30 \%$ for samples stored at $45^{\circ} \mathrm{C}$ and a loss of $10-15 \%$ for samples stored at $20{ }^{\circ} \mathrm{C}$. Based on these results and using an Arrhenius equation for prediction, predicted yearly losses of activity at $-20{ }^{\circ} \mathrm{C}$ for either of these two preparations would be less than $0 \cdot 1 \%$.

For each of the three ampouled preparations, estimates by in vitro bioassays for the ATD samples and the samples stored continuously at $-20{ }^{\circ} \mathrm{C}$ did not differ significantly from one another relative to the intra-assay and intra-laboratory variability.

Estimates by immunoassays and receptor assays for ATD samples of each of the three ampouled preparations differed significantly between different assay systems, although estimated losses of activity were generally less than those obtained by in vivo bioassays except for 92/642, and not detectable by some assay systems. This indicates that these various assay systems show significantly different specificities for the degraded and less biologically active FSH.

On the basis of these results, each of the ampouled preparations appears to be sufficiently stable to serve as a standard.

\section{Discussion}

Separate standards for FSH from pituitaries and from post-menopausal urine were established in the 1960s on the basis that gonadotrophins extracted from these two sources differed from one another, although the unitages for each were derived from a common preparation. The ISU is the standard in current use for calibration by bioassay of therapeutic products. However, the development and therapeutic use of high purity and 
Table 9 Unweighted laboratory geometric mean estimates (gms) for the FSH content of the IS human rFSH in ampoules coded 92/642, and of a preparation of $\mathrm{rFSH}$ in ampoules coded $92 / 510$ obtained by immunoassay, expressed as IU of the IS for human urinary FSH in ampoules coded 92/512 per ampoule and of the preparation of rFSH in ampoules coded 92/510 expressed as IU of the IS for human rFSH per ampoule. The number of independent estimates contributing to each mean estimate is shown in parentheses beside the laboratory code where there were the same number of estimates for each preparation, and otherwise beside the mean estimate. The $95 \%$ fiducial limits for the unweighted geometric mean of laboratory mean estimates is based on the variance of the logarithms of the estimates combined

\section{Laboratory number}

Two-site immunoassays

$1(2)$

$2(2)$

$5(3)$

$6 \mathrm{~A}(2)$

$6 \mathrm{~B}(2)$

7 (4)

$9(2)$

$15(4)$

20

$21(2)$

$27(2)$

28A (3)

28B (3)

$29 \mathrm{~A}(2)$

29B (2)

30A (2)

30B

Geometric mean of laboratory gms

Omitting estimates marked*

\section{One-site immunoassays}

\section{$2(2)$}

$3 \mathrm{~A}(2)$

$3 \mathrm{~B}(3)$

$6(2)$

$8(2)$

$17(2)$

$19(2)$

22 (4)

28 (3)

Geometric mean of laboratory gms

Omitting estimates marked*

One-site and two-site immunoassays Geometric mean of laboratory gms Omitting estimates marked* $\mathbf{9 2 / 6 4 2}$ (as IU of 92/512)

\begin{tabular}{l}
\hline \\
189 \\
177 \\
173 \\
199 \\
174 \\
185 \\
161 \\
180 \\
196 \\
174 \\
172 \\
183 \\
169 \\
189 \\
$129^{*}$ \\
$142^{*}(5)$ \\
173 (16) $163-184$ \\
180 (14) $174-186$
\end{tabular}

92/510 (as IU of 92/512)

92/510 (as IU of 92/642)

$\begin{array}{lcl}173 & 123 & 98 \\ 166 & 108 & 90 \\ 174 & 109 & 87 \\ 159 & 92 & 80 \\ 101^{*} & 55^{*} & 76^{*} \\ 166 & 105 & 87 \\ 155 & 94 & 84 \\ 163 & 100 & 85 \\ 163 & 111 & 94 \\ 156(9) 137-177 & 98(9) 82-117 & 86 \text { (9) } 81-92 \\ 165(8) 159-170 & 105 \text { (8) } 97-113 & 88 \text { (8) } 83-93 \\ & & \\ 167(25) 157-177 & 104(24) 97-112 & 86(24) 83-88 \\ 174(22) 169-179 & 109(21) 105-113 & 86(21) 84-89\end{array}$

rDNA-derived preparations of FSH has led to a review of standards for therapeutic FSH preparations. This study has provided comparisons of the ISU with candidate preparations of high purity urinary FSH and of $\mathrm{rFSH}$.

\section{Calibration of candidate IS by in vivo bioassay}

Some 25 comparisons by in vivo bioassay for each of the candidate preparations with the ISU gave assays which were broadly statistically valid and estimates of relative potency which, for each comparison, were homogeneous, with a maximum of two heterogeneous estimates for any comparison. Thus, for each candidate standard, valid calibration by in vivo bioassay in terms of the ISU was obtained, giving for 92/512 a weighted geometric mean (95\% fiducial limits) of 121 (113-128) IU per ampoule, for 92/642 a weighted geometric mean of 138 (131-145) IU per ampoule, and for $92 / 510$ a weighted geometric mean of 95 (90-100) IU per ampoule.

These results are consistent with studies which have found that the in vivo biological activity of rFSH (De Boer \& Mannaerts 1990, Galway et al. 1990), and its pharmacokinetic and pharmacodynamic characteristics (Porchet \& le Cotonnec 1994, Weinbauer et al. 1994) are similar to those of urinary FSH, and that $\mathrm{rFSH}$ gave a doseresponse curve in the Steelman-Pohley bioassay which 


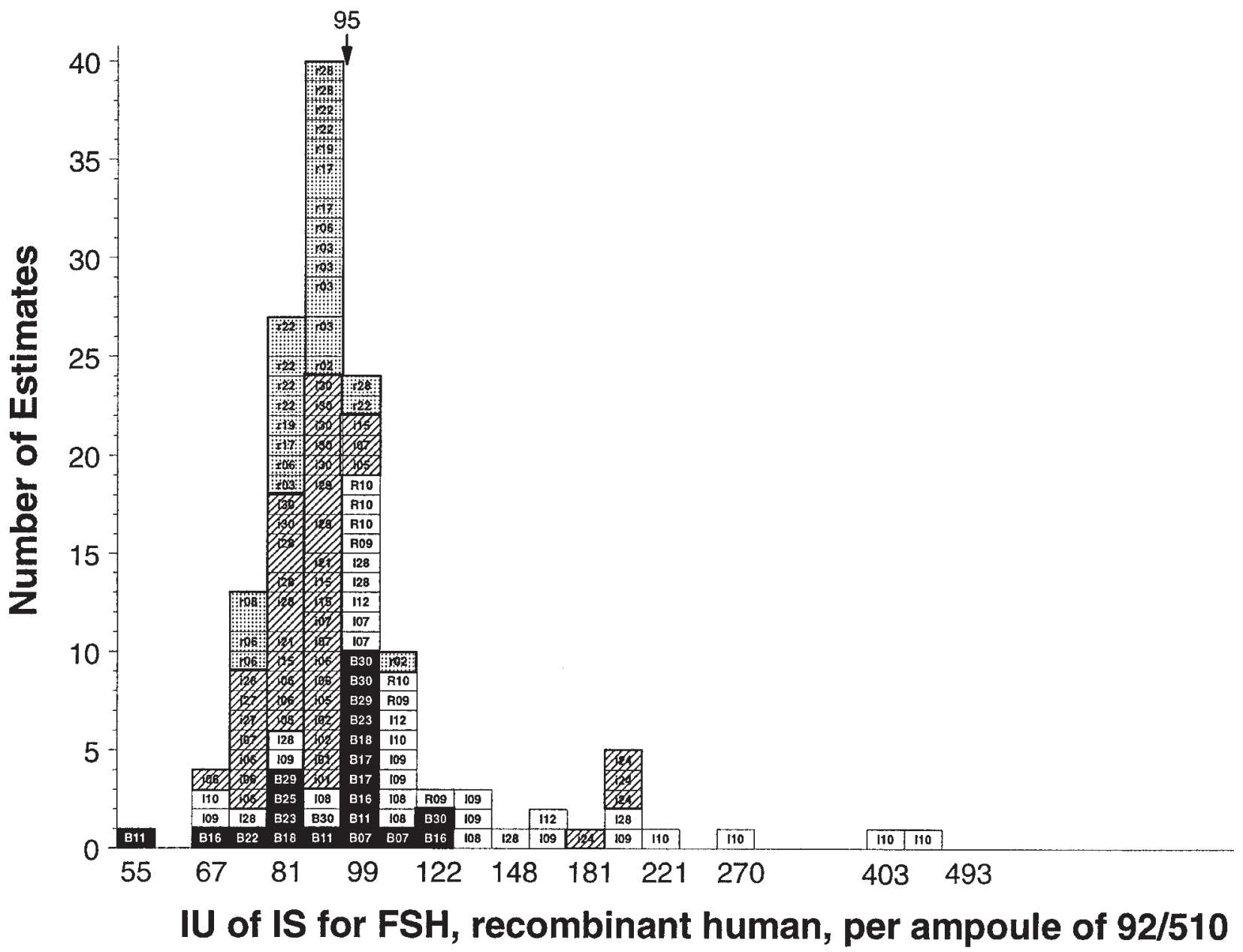

Figure 6 Individual estimates of the FSH content for ampoules coded 92/510 expressed as IU of the IS for FSH (in ampoules coded 92/642) per ampoule of 92/642. Codes and shading as in Fig. 1. The arrowed number shows the weighted geometric mean of the estimates by bioassay.

was parallel to a menotrophin standard and linear in the same part of the curve. Thus clinical protocols and dosage regimens similar to those for urinary FSH have been proposed for rFSH (Porchet \& le Cotonnec 1994).

Since $\mathrm{rFSH}$ preparations appear to behave overall in a manner similar to preparations derived from urinary sources and in the interests of maintaining continuity of unitage from one standard to another and hence from one therapeutic product to another the IS for rFSH was calibrated directly in terms of the IS for menotrophin.

\section{Comparisons by in vitro bioassay}

In the present study estimates of activity by in vitro bioassays showed significant differences between laboratories. The variability of estimates obtained from in vitro bioassays may reflect the selectivity or specificity of these assays which in general select only one action of
FSH as a measurable end point. A number of studies have compared in vitro bioactivity, or structural features of $\mathrm{rFSH}$ with preparations derived from natural sources. Thus rFSH has been found to have similar activity to natural FSH in some in vitro assay systems (Galway et al. 1990, Mannaerts et al. 1991, Mason et al. 1993) and to be more potent in other assay systems (Flack et al. 1994, Lambert et al. 1995). Results obtained here using receptor binding assays were more consistent between laboratories than those from in vitro bioassays but there were a limited number of systems used. However, it may be that receptor binding and signal transduction are dissociable functions which require separate sites of the FSH molecule (Valove et al. 1994). Thus while receptor binding may require properties of FSH which are consistent between different preparations, in vitro bioassays may determine properties which are variable between different preparations. 


\section{Comparison by immunoassay}

Estimates of activity by immunoassay differed consistently and significantly between laboratories indicating that immunoassays may have different molecular specificities. Numerous publications have highlighted the variability between different immunoassay systems (Seth et al. 1989, Taylor et al. 1994) which may arise from differences in specificity or affinity of different antibodies for standard materials or test samples (Seth et al. 1989, Jockenhovel et al. 1990), different standard preparations used in different assays and different reference preparations used for calibrating kit standards. Thus even if a highly purified standard were used for calibration of diagnostic immunoassay some variability in estimates of activity in clinical samples could be expected.

The in vivo bioassays, which are specified by the Pharmacopoeia for calibration of therapeutic products, did not show consistent inter-laboratory differences between estimates for any of the comparisons considered in this study. However, estimates obtained by in vitro bioassay and immunoassay varied depending on the assay system and on the preparation used as standard. Some of these latter assay systems appeared to distinguish between $\mathrm{rFSH}$ preparations, for example. The ratio of biological activity to immunological activity for in vivo bioassays was about half that for in vitro bioassays for some comparisons. Thus assays which do not take account of biological clearance in the same way may give significantly different estimates of biological activity. The inter-laboratory variabilities for both in vitro bioassays and immunoassays suggest that the ratio of biological activity to immunological activity is not a stable parameter.

\section{Conclusions}

The standards describe here enable calibration of therapeutic products derived from highly purified urinary and from pure $\mathrm{rFSH}$ on the basis of the underlying principle of comparing 'like with like'. There is a discontinuity of unitage between the calibration of all the candidate standards by immunoassays and by in vivo biological assays. Studies of the calibration of FSH standards for diagnostic immunoassays will be considered separately.

Based on the results of this study the Expert Committee on Biological Standardization of WHO established the preparation in ampoules coded $92 / 512$ as the IS for urinary FSH (urofollitropin) with an assigned potency of $121 \mathrm{IU}$ per ampoule on the basis of its calibration by in vivo bioassay in terms of the ISU.

The preparation coded $92 / 642$ was established as the IS for human $\mathrm{rFSH}$ and assigned a value of $138 \mathrm{IU}$ per ampoule on the basis of its calibration by in vivo bioassay in terms of the ISU. This unitage maintains a continuity of activity with ISU used to calibrate urinary-derived therapeutic materials. The unitages assigned to $92 / 512$ and $92 / 642$ are also consistent with their direct calibration by in vivo bioassay in terms of one another. The ISU was retained as a standard for bioassay of urinary FSH and LH (menotrophin). All of the above standards were deemed suitably stable to act as WHO IS. They have been calibrated by the in vivo bioassay and are intended as standards for the calibration of such assays.

These standards are available from the National Institute for Biological Standards and Control (Fax +(44) 1707 646977; Tel +(44) 1707 646399; email standards@nibsc. ac.uk; mail NIBSC, PO Box 1193, Blanche Lane, Potters Bar, Hertfordshire EN6 3QG, UK).

\section{Acknowledgements}

Grateful acknowledgements are due to the participants in this study, to the Ares-Serono Group (Geneva) and to Organon International (The Netherlands) for generous provision of candidate standards for FSH, to Dr Adam Balen for provision of serum from pre-menopausal women, to Dr P Dawson and staff of the Standards Processing Division at NIBSC for distribution of materials into ampoules, to Mrs J Longley and Mr B Rafferty for preliminary characterisation of candidate standards, to Mrs Gill Creeber for data entry and to Ms Margaret Lewis and Mrs Angela Witt for preparation of the manuscript and tables.

\section{References}

Amoresano A, Siciliano R, Orru S, Napoleoni R, Altarocca V, De Luca E, Sirna A \& Pucci P 1996 Structural characterisation of human recombinant glycohormones follitropin, lutropin and choriogonadotropin expressed in Chinese hamster cells. European Journal of Biochemistry 242 608-618.

British Pharmacopoeia 1988 Biological Assay of Menotrophin. XIV. C4.

Cheng KW 1975 A radioreceptor assay for follicle-stimulating hormone. Journal of Clinical Endocrinology and Metabolism $\mathbf{4 1}$ 581-589.

De Boer W \& Mannaerts B 1990 Recombinant follicle stimulating hormone II. Biochemical and biological characteristics. In From Clone to Clinic, pp 253-259. Eds DJA Crommelin \& H Schellekens. Netherlands: Kluwer Academic Publishers.

Devroey P, Van Steirteghem A, Mannaerts B \& Coelingh Bennink H 1992 Successful in vivo fertilisation and embryo transfer after treatment with recombinant human FSH. Lancet 3391170.

European Pharmacopoeia 1986 Menotropinum. Second Part II. Tenth Fascicule. Monograph 508.

Flack MR, Bennet AP, Froehlich J, Anasti JN \& Nisula BC 1994 Increased biological activity due to basic isoforms in recombinant human follicle-stimulating hormone produced in human cell line. Journal of Clinical Endocrinology and Metabolism 79 756-760.

Gaines-Das RE \& Tydeman M 1982 Iterative weighted regression analysis of logit responses: A computer program for analysis of bioassays and immunoassays. Computer Programs in Biomedicine $\mathbf{1 5}$ $13-22$. 
Galway AB, Hsueh AJ, Keene JL, Yamoto M, Fauser BC \& Boime 1990 In vitro and in vivo bioactivity of recombinant human follicle stimulating hormone and partially deglycosylated variants secreted by transfected cell lines. Endocrinology 127 93-100.

Giudice E, Crisci C, Eshkol A \& Papoian R 1994 Composition of commercial gonadotrophin preparations extracted from post-menopausal urine: characterisation of non-gonadotrophin proteins. Human Reproduction 9 2291-2299.

Gudermann T, Brockman H, Simoni M, Gromoll J \& Nieschlag E 1994 In vitro bioassay for human serum follicle-stimulating hormone (FSH) based on L cells transfected with recombinant rat FSH receptor: validation of a model system. Endocrinology 135 2204-2213.

Guillou F, Martinat N \& Combarnous Y 1986 Study of the superactivity of equine follicle-stimulating hormone in in vitro stimulation of rat Sertoli cells. Biochimica et Biophysica Acta $\mathbf{8 8 7}$ 196-203.

Hard K, Mekking A, Damm JBL, Kamerling JP, De Boer W, Wijnands RA \& Vliegnethart FG 1990 Isolation and structure determination of the intact sialylated $\mathrm{N}$-linked carbohydrate chains of recombinant human follitropin expressed in Chinese hamster ovary cells. European Journal of Biochemistry 193 263-271.

Harris SD, Anobile CJ, McLoughlin JD, Mitchell R, Lambert A \& Robertson WR 1996 Internal complexity of the oligosaccharide chains of recombinant human follicle stimulating hormone (Puregon, Org 32489); a comparison with Metrodin and Metrodin-HP. Molecular Human Reproduction 2 807-811.

Howles CM, Loumaye E, Giroud D \& Luyet G 1994 Multiple follicular development and ovarian steroidogenesis following subcutaneous administration of a highly purified urinary FSH preparation in pituitary desensitized women undergoing IVF: a multicentre European phase III study. Human Reproduction 9 424-430.

Hugues JN 1994 Metrodin HP: a new highly purified FSH. Contraception Fertility and Sex 22 402-404.

Jockenhovel F, Khan SA \& Nieschlag E 1990 Varying dose-response characteristics of different immunoassays and an in vitro bioassay for FSH are responsible for changing ratios of biologically active to immunologically active FSH. Journal of Endocrinology 127 523-532.

Lambert A, Rodgers M, Mitchell R, Wood AM, Wardle C, Hilton B \& Robertson WR 1995 In vitro biopotency and glycoform distribution of recombinant human follicle stimulating hormone (Org 32489), Metrodin and Metrodin HP. Human Reproduction 10 1928-1935.

Mannaerts B, De Leeuw R, Geelen J, Van Ravestein A, Van Wezenbeek P, Schuurs A \& Kloosterboer H 1991 Comparative in vitro and in vivo studies on the biological characteristics of recombinant human follicle-stimulating hormone. Endocrinology 129 2623-2630.

Mason HD, Mannaerts B, De Leeuw R, Willis DS \& Franks S 1993 Effects of recombinant follicle stimulating hormone on cultured human granulosa cells: comparison with urinary gonadotrophins and actions in pre-ovulatory follicles. Human Reproduction 8 1823-1827.

Miro F, Smyth CD \& Hillier SG 1991 Development-related effects of recombinant activin on steroid synthesis in rat granulosa cells. Endocrinology 129 3388-3394.

Padmanabhan V, Chappel SC \& Beitins IE 1987 an improved in vitro bioassay for follicle-stimulating hormone (FSH): suitable for measurement of FSH in unextracted human serum. Endocrinology 121 1089-1098.
Porchet HC \& le Cotonnec J 1994 Pharmacokinetic and pharmacodynamic characteristics of recombinant human follicle-stimulating hormone. Assisted Reproduction Reviews 4 110-117.

Raab GM 1981 Estimation of a variance function with application to immunoassay. Applied Statistics 30 32-40.

Seth J, Hanning I, Bacon RRA \& Hunter WM 1989 Progress and problems in immunoassays for serum pituitary gonadotrophins: evidence from the UK external quality assessment schemes (EQAS) 1980-1988. Clinica Chimica Acta 186 67-82.

Steelman SL \& Pohley FM 1953 Assay of follicle-stimulating hormone based on augmentation with human chorionic gonadotropin. Endocrinology 53 604-616.

Storring PL 1988 The role of biological assays in evaluating purified peptide and protein products. In Therapeutic Peptides and Proteins: Assessing New Technologies, pp 119-134. Cold Spring Harbor: Cold Spring Harbor Laboratory.

Storring PL, Dixon H \& Bangham DR 1976 The first international standard for human urinary FSH and for human urinary LH (ICSH), for bioassay. Acta Endocrinologica 83 700-710.

Taylor AE, Khoury RH \& Crowley WRJ 1994 A comparison of 13 different immunometric assay kits for gonadotrophins: implications for clinical investigation. Journal of Clinical Endocrinology and Metabolism 79 240-247.

Tilly JL, Aihara T, Nishimori K, Jia X, Billig H, Kowalski KI, Perlas EA \& Hsueh AJW 1992 Expression of recombinant human follicle-stimulating hormone receptor: species-specific ligand binding, signal transduction, and identification of multiple ovarian messenger ribonucleic acid transcripts. Endocrinology 131 799-806.

Valove FM, Finch C, Anasti JN, Froelich J \& Flack MR 1994 Receptor binding and signal transduction are dissociable functions requiring different sites on follicle-stimulating hormone. Endocrinology 135 2657-2661.

Van Wezenbeek P, Draaijer J, Van Meel F \& Olijve W 1990 Recombinant follicle stimulating hormone I. Construction, selection and characterisation of a cell line. In From Clone to Clinic, pp 245-251. Eds DJA Crommelin \& H Schellekens. Netherlands: Kluwer Academic Publishers.

Weinbauer GF, Simoni M, Hutchison JS \& Nieschlag ES 1994 Pharmacokinetics and pharmacodynamics of recombinant and urinary human FSH in the male monkey (Macaca fascicularis). Journal of Endocrinology 141 113-121.

WHO Expert Committee on Biological Standardization 1964 Human Menopausal Gonadotrophins. WHO Technical Report Series No. 293.

WHO Expert Committee on Biological Standardization 1975 International Standards and International Reference Preparations. 1. Human Urinary Gonadotrophins. WHO Technical Report Series No. 565, pp 6-7.

WHO Expert Committee on Biological Standardization 1989 Follicle Stimulating Hormone and Luteinizing Hormone, Urinary. WHO Technical Report Series No. 39, p 23.

WHO Expert Committee on Biological Standardization 1994 Follicle Stimulating Hormone and Luteinizing Hormone, Human, Urinary. WHO Technical Report Series No. 848, p 19.

Received 8 December 1997 Accepted 16 February 1998 